\title{
Cucker-Smale Flocking Under Asynchronous Update Dynamics
}

\author{
Jing Ma and Edmund M-K Lai \\ Department of Information Technology \& Software Engineering \\ Auckland University of Technology \\ Auckland, New Zealand \\ Email:\{jing.ma, edmund.lai\}@aut.ac.nz
}

\begin{abstract}
The effects of asynchronous updating of the state of the agents in a Cucker-Smale flocking model is considered in this paper. The study of asynchronous update is important because in practical implementations the agents typically have internal clocks that are not synchronized. We considered how asynchronous update will affect the time it takes to achieve flocking (flocking time) as well as how close the agents in the flock are moving (flock diameter). These factors were largely ignored in most previous works as achieving asymptotic convergence was their main focus. Furthermore, previous simulations typically assume that the agents move with the same speed. We considered the effects of achieving consensus of both the speed and the heading. Through computer simulations, we showed that both the flocking time and flock diameter increase significantly with asynchronicity. Results also showed that the diameter of the flock is substantially larger when the agents start with different speeds. These results should be taken into account when designing flocking agents in practice.
\end{abstract}

Keywords: Asynchronous update, Cucker-Smale, Flocking

\section{Introduction}

Flocking is a collective behaviour where individual autonomous agents use only limited information to selforganize into a state of motion consensus, starting from a disordered initial state. From the distributed system point of view, flocking is achieved when individual agents reached consensus on their velocity. Consensus has been a very active area of research in the past decade [1]-[4]. The type of agents are typically either first-order [5], [6] or second-order [3], [7], [8]. The two most studied models of flocking - the Vicsek model [9] and the Cucker-Smale model [10], are both second order models. These two models are similar in the sense that an agent's velocity is adjusted depending on a weighted average of the velocities of the agents in some neighbourhood. The Cucker-Smale model has the added advantage that it is a continuous-time model and its convergence has been proven based only on the initial state and a coupling parameter.

Most of the researches on flocking and consensus have been assumed that the agents either update their states continuously for continuous-time models or at the same time instants (i.e. synchronously) for discrete-time models. In practice, the internal clocks of the agent are rarely synchronized. There has been some researches in the asynchronous consensus problem, where the internal clocks of the agents are not synchronized. The types of asynchronous update include overlapping, sequential, parallel and blocksequential updates [11]. The convergence of asynchronous consensus was first reported in [12], [13]. De Castro et al. [14] studied the asynchronous consensus problem for discrete-time multi-agent systems with a fixed communication topology. In 2005, Fang et al. studied the consensus of asynchronous discrete-time multi-agent systems, with various communication patterns [15]. Asynchronous consensus control for a continuous-time multi-agent system with switching topology is studied in [16]. The continuoustime system was transformed into its equivalent discretetime system, and a distributed consensus algorithm was proposed to address problems such as time-varying communication delays. Meanwhile, an asynchronous Vicsek model has been studied in which each agent independently updates its heading at times determined by its own clock [17].

In all the above cited works, the focus is on the asymptotic convergence properties of some consensus protocols or flocking model under various conditions. The time it takes to achieve flocking or consensus and how asynchronous update affects it has not been studied. Another measure of a flock is how spread out in space the flock is, which we shall call the flock diameter, has also not been considered. Furthermore, for most of the simulation results with the two flocking models, the speed of the agents is typically assumed to be the same. Thus only the consensus of the heading is considered. It is not clear whether the consensus of both speed and heading will affect the flocking time.

The main objective of this paper is to present simulation results concerning the flocking time and flock diameter for the discrete-time version of the Cucker-Smale model with asynchronous updates. A secondary objective is to present results when both the speed and heading needs to be aligned. These results shall be useful for practical design of systems of flocking agents. A brief introduction to the definition of flocking and the Cucker-Smale flocking model is given in Section 2. In Section 3, the method we used to simulate asynchronous updates and the simulation results are presented. 


\section{Cucker-Smale Model and Flocking}

\subsection{Definition of Flocking}

Flocking is said to be achieved when the following two conditions are satisfied.

1) The velocities of the agents are aligned. Mathematically, for an arbitrarily small $\delta>0$, this condition is described by

$$
\left|v_{i}-v_{j}\right| \leq \delta
$$

for all $i, j \in[1, N], i \neq j$.

A more convenient measure of velocity alignment is the average normalized velocity. It is defined as

$$
v_{a}=\frac{\left|\sum_{i=1}^{N} v_{i}\right|}{\sum_{i=1}^{N}\left|v_{i}\right|}
$$

The alignment condition can be expressed as $\mid 1-$ $v_{a} \mid<\delta^{\prime}$ for some small $\delta^{\prime}>0$.

In a two-dimensional system, velocity alignment requires the consensus of both the speed $s_{i}$ and the heading $\theta_{i}$. That is,

$$
\begin{aligned}
& \left|\theta_{i}(t)-\theta_{j}(t)\right| \leq \delta_{\theta} \\
& \left|s_{i}(t)-s_{j}(t)\right| \mid \leq \delta_{s} \quad \forall i, j=1,2, \ldots, N .
\end{aligned}
$$

for some arbitrarily small $\delta_{\theta}$ and $\delta_{s}$.

2) The agents are cohesive. That is, the distance between any two agents is bounded by some finite positive constant $\epsilon$. That is,

$$
0<\sup _{t \geq T_{f}}\left\|p_{i}-p_{j}\right\|<\epsilon
$$

for all $1 \leq i, j \leq N$.

With any flocking model, we are interested in two performance measures. They are the flocking time and flocking diameter. The flocking time is the time it takes for the velocities of the agents to be aligned. Using the notations above, it is defined as $T_{f}=\inf \left\{T:\left|v_{i}-v_{j}\right| \leq \delta\right\}$. The flocking diameter is the distance between any two agents that are furthest apart.

\subsection{Cucker-Smale Model}

The Cucker-Smale model was first proposed in [10] to capture the emergence of flocking behaviour of a group of $N$ autonomous agents in both continuous and discrete time. The state of agent $i(1 \leq i \leq N)$ is described by its position $p_{i}$ and velocity $v_{i}$ in the following way:

$$
\left\{\begin{array}{l}
\dot{p}_{i}=v_{i} \\
\dot{v}_{i}=\frac{1}{N} \sum_{j=1}^{N} \psi\left(\left\|p_{j}-p_{i}\right\|\right)\left(v_{j}-v_{i}\right)
\end{array}\right.
$$

where $\psi$ is a positive decreasing function that quantifies the influence of robot $j$ on the velocity of robot $i$. A function that has been used extensively is given by

$$
\psi\left(\left\|p_{j}-p_{i}\right\|\right)=\frac{1}{\left(1+\left\|p_{j}-p_{i}\right\|^{2}\right)^{\beta}} .
$$

In [10], it has been proven that flocking emerges unconditionally if $\beta<1 / 2$. For $\beta \geq 1 / 2$, however, flocking is conditional on the initial state of the system.

The Cucker-Smale model is a continuous-time model which makes it amenable to mathematical analysis. However, when it is implemented on real agents, velocity changes have to be made at regular discrete intervals instead of in a continuous fashion. In discrete time, (5) is given by

$$
\Delta v_{i}\left(t_{(k+1)}\right)=\frac{1}{N} \sum_{j=1}^{N} a_{i j}\left(t_{k}\right)\left(v_{j}\left(t_{k}\right)-v_{i}\left(t_{k}\right)\right),
$$

for $i \in 1,2, \ldots, N$, where $a_{i j}\left(t_{k}\right)=\psi\left(\left\|p_{j}-p_{i}\right\|\right)$ at the time instant $t_{k}$. Most simulation results of flocking are obtained assuming that all the agents update their states at the same time using the latest values of the neighbour's states. That is, the state of the agents is changed at time instants $t_{1}, t_{2}, \ldots, t_{k}, t_{k+1}, \ldots$ instantaneously. This basically assumes that the internal clocks of all the agents are synchronized.

If the internal clocks of the agents are not synchronized, then they will not be updating their states at the same time instant. Figure 1 shows an example of asynchronous update; some agents update at the same time instant while others do not.

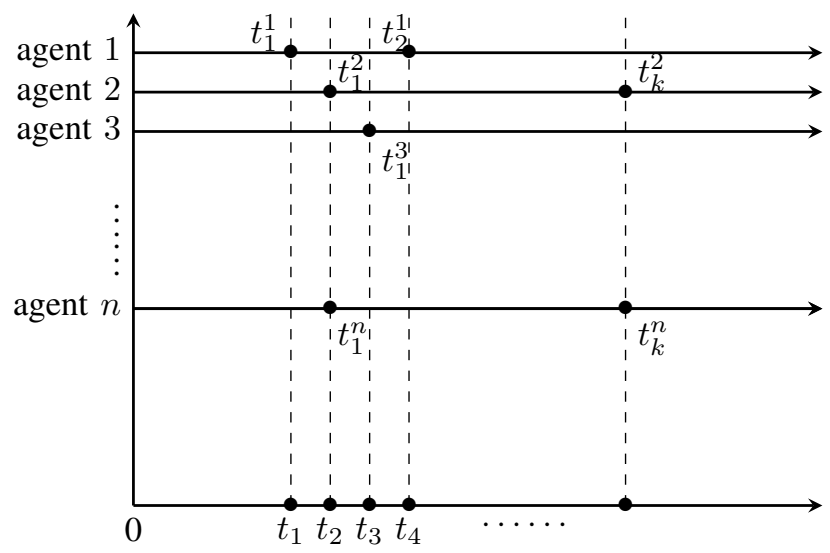

Figure 1. Updating Times in Asynchronous Dynamic System

\section{Simulating Asynchronous Update}

\subsection{Methodology}

We shall investigate the effects of asynchronous update to the flocking time and diameter through computer simulations. Assume that each agent updates its state every $T_{p}$ seconds. Then, in the synchronous case, the state of 
every agent is updated at time instants $T_{p}, 2 T_{p}, 3 T_{p}, \cdots$. To simulate asynchronous update, every $T_{p}$ second interval is divided into $\alpha$ equal time periods. Each of the $N$ agents in the system is random assigned to one of the $\alpha$ subgroups, with subgroup $i$ containing $N_{i}>0$ agents. The agents in each subgroup are updated at the same time. Consider the time interval $\left[0, T_{p}\right]$. Agents in subgroup 1 will be updated at time $T_{p} / \alpha$. Those in subgroup $i$ are updated at $i T_{p} / \alpha$ and those in subgroup $\alpha$ at $T_{p}$. This process is repeated every $T_{p}$ seconds. The average velocity is computed after each subgroup updates to see if the flocking condition is satisfied.

\subsection{Results}

In our experiments, the agents move in an infinitely large two-dimensional space. Consequently, they will not encounter any boundary. The agents initially move in a random direction with either the same speed or different speeds. It has been shown in [10] that the flocking will be achieved if $\beta<1 / 2$. Hence, we set $\beta$ to $1 / 4$ to ensure that flocking occurs. For convenience, distances shall be dimensionless and time is in seconds. The system is considered to be in a flocking state when $v_{a} \geq 0.99$. The flock diameter is measured when this condition occurs. The initial position of the agents is randomly chosen within a $4 \times 4$ square. The initial heading is uniformly random in $[0,2 \pi)$. Each simulation scenario is repeated 20 times with different random initial values. The results presented are the average values.

\subsubsection{Synchronous Update With Different Speed.}

We shall first consider synchronous update. In the first set of simulations, the agents move with the same constant speed of 0.5 per second. The number of agents $N$ varies from 10 to 50. This provides us with the basis for comparison with existing literature. In the second set of simulations, the agents move with different initial speed and heading. An agent's speed is initialized to within the range of $0.5 \pm 0.05$ per second.

Figure 2 shows the flocking times for both sets of simulations. For the same flock size, the flocking time required for agents with different initial speed is longer than when the agents start with the same speed. This implies that it takes longer to reach consensus of both speed and heading that with heading alone. Furthermore, in both cases, doubling the flock size $N$ increases the flocking time by $30-40 \%$.

Figures 3 and 4 show the convergence behaviour reflected by the average normalized velocity for the two sets of simulations respectively. As expected, the initial convergence is slower for agents with different initial speeds.

\subsubsection{Effects of Asynchronous Update.}

The method described in Section 3.1 is used to simulate asynchronous update. By varying the number of subgroups, we can vary the degree of synchronicity from fully synchronous to almost completely asynchronous.

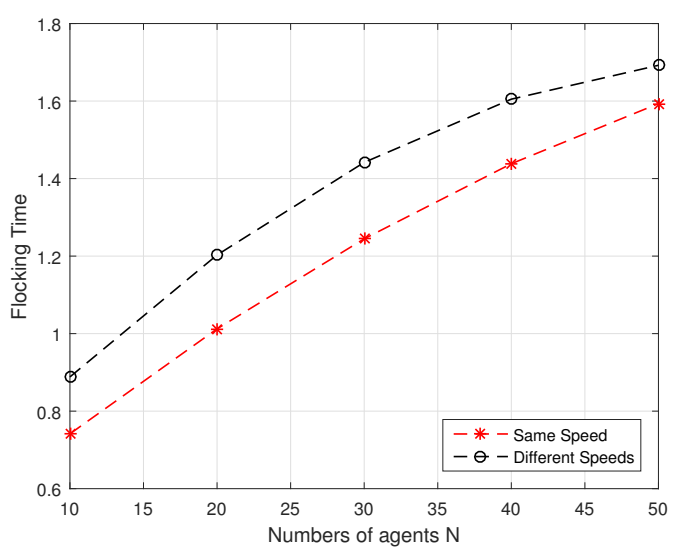

Figure 2. Flocking Time with Synchronous Updating

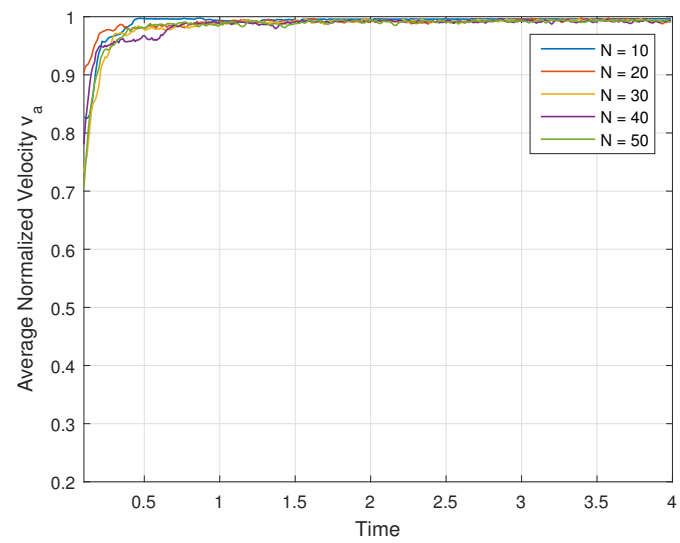

Figure 3. Consensus on velocity with synchronous updating for same speed

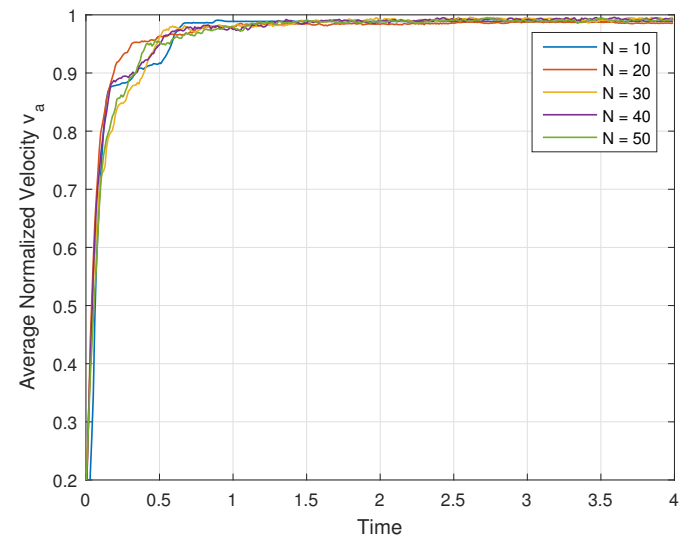

Figure 4. Consensus on velocity with synchronous updating for different initial speeds 


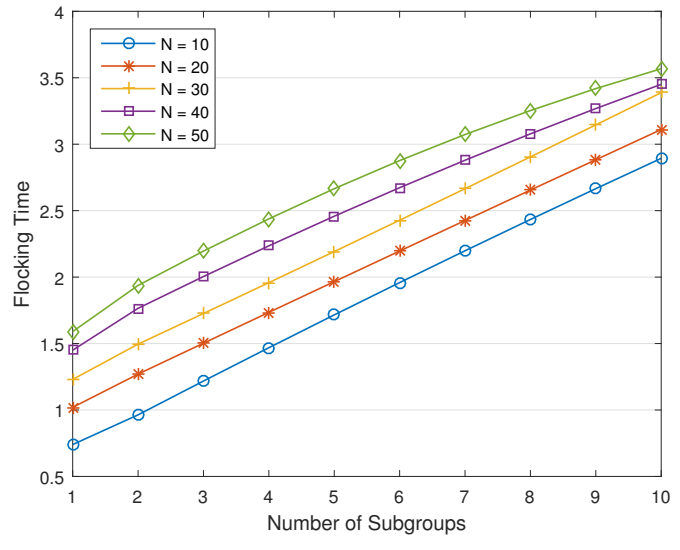

Figure 5. Flocking time with asynchronous updating

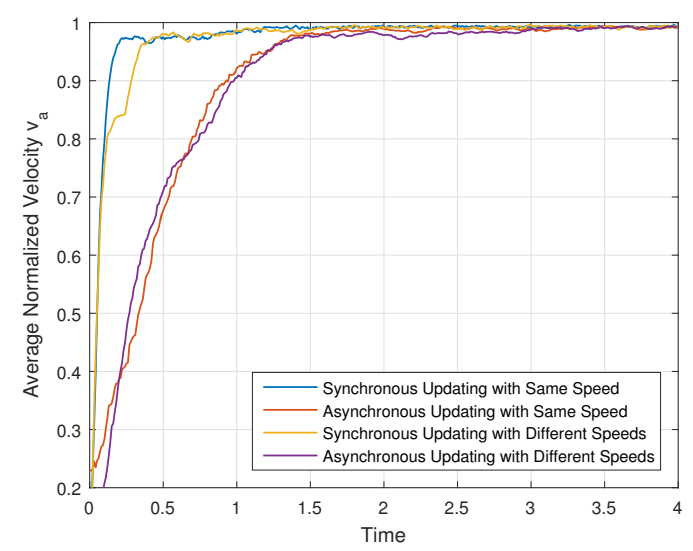

Figure 6. Convergence behaviours of synchronous and asynchronous updating for $N=30$.

The flocking time results are shown in Figure 5 for different number of subgroups and different $N$. With this set of simulations, the initial speed of the agents is the same. The graphs show that flocking time increases approximate linearly with the degree of asynchronism for $N$ up to 30 , with a slope of around 0.22 . For instance, for $N=10$ divided into 10 subgroups of 10 agents, the flocking time is four times that of synchronized updates (1 subgroup). As $N$ increases, the curves become more nonlinear. This is due to the fact that even with 10 subgroups, there are still quite a number of agents in each subgroup that are updated at the same time.

Figure 6 compares how the average normalized velocity $v_{a}$ changes with time for $N=30$ under synchronous and asynchronous updating. In the case of asynchronous updating, 5 subgroups are used. These results show that it takes much longer for the system to converge to an average normalized velocity above 0.9 with asynchronous updating. Interestingly, whether the agents start with different speed or the same speed makes little difference for asynchronous update. But this is not the case for synchronous update.
Table 1 shows the flock diameters when flocking has been achieved. The columns labelled "SS" are for agents starting with the same speed. The columns labelled "DS" are for agents starting with different speeds. First, these results show that the flock diameters increase substantially with the degree of asynchronicity regardless of whether the agents start with same speed or not. Second, for the same degree of asynchronicity, agents starting with different speeds eventually flock further apart compared with those that start with the same speeds. The reason for these effects is not apparent and therefore require further research.

\section{Conclusions}

In this paper, we confirmed that flocking can be achieved with the Cucker-Smale model when consensus on both the speed and heading is required. Under asychronous updating dynamics, the time it takes to achieve flocking is substantially increased when compared to synchronous update. This means that in practical implementation of such systems, a longer flocking time should be taken into account. A somewhat surprising result is that the flock diameters are significantly larger with asynchronous update. Furthermore, systems with agents starting with different speeds eventually flock further apart than the ones with agents starting with the same speed. Further investigation will be needed in order to find out why this is the case.

\section{References}

[1] V. Blondel, J.M. Hendrickx, A. Olshevsky and J. Tsitsiklis. Convergence in multiagent coordination, consensus, and flocking. IEEE Conference on Decision and Control, 44(3), 2296, 2005.

[2] W. Dong and J.A. Farrell. Consensus of multiple nonholonomic systems. 47th IEEE Conference on Decision and Control, 22702275, 2008.

[3] W. Yu, G. Chen and M. Cao. Some necessary and sufficient conditions for second-order consensus in multi-agent dynamical systems. Automatica, 46(6):1089-1095, 2010.

[4] W. Yu, L. Zhou, X. Yu, J. Lü and R. Lu. Consensus in multiagent systems with second-order dynamics and sampled data. IEEE Transactions on Industrial Informatics, 9(4):2137-2416, 2013.

[5] A. Jadbabaie, J. Lin, and A.S. Morse. Coordination of groups of mobile autonomous agents using nearest neighbor rules. IEEE Transactions on Automatic Control, 48(6), 985-1001, 2003.

[6] W. Ren and R.W. Beard. Consensus seeking in multiagent systems under dynamically changing interaction topologies. IEEE Transactions on Automatic Control, 50(5), 655-661, 2005.

[7] W. Ren and E. Atkins. Second-order consensus protocols in multiple vehicle systems with local interactions. AIAA Guidance, Navigation, and Control Conference and Exhibit,6238, 2005.

[8] W. Yu, G. Chen, M. Cao, and J. Kurths. Second-order consensus for multi-agent systems with directed topologies and nonlinear dynamics. IEEE Transactions on Systems, Man, and Cybernetics, Part B (Cybernetics), 40(3), 881-891, 2010.

[9] T. Vicsek, A. Czirók, E. Ben-Jacob, I. Cohen and O. Shochet. Novel type of phase transition in a system of self-driven particles. Physical review letters, 75(6), 1226, 1995.

[10] F. Cucker and S. Smale. Emergent behaviour in flocks. IEEE Transactions on Automatic Control, 52(5):852-862, 2007. 
TABLE 1. Flock DIAMETERS WITH ASYNCHRONOUS UPDATING FOR SAME INITIAL SPEED (SS) AND DIFFERENT INITIAL SPEED (DS).

\begin{tabular}{|c|c|c|c|c|c|c|c|c|c|c|}
\hline \multirow{2}{*}{ Number of Groups } & \multicolumn{2}{|c|}{$\mathrm{N}=10$} & \multicolumn{2}{c|}{$\mathrm{N}=20$} & \multicolumn{2}{c|}{$\mathrm{N}=30$} & \multicolumn{2}{c|}{$\mathrm{N}=40$} & \multicolumn{2}{c|}{$\mathrm{N}=50$} \\
\cline { 2 - 12 } & $\mathrm{SS}$ & $\mathrm{DS}$ & $\mathrm{SS}$ & $\mathrm{DS}$ & $\mathrm{SS}$ & $\mathrm{DS}$ & $\mathrm{SS}$ & $\mathrm{DS}$ & $\mathrm{SS}$ & $\mathrm{DS}$ \\
\hline 1 & 1.2294 & 1.5268 & 1.2244 & 1.5347 & 1.2200 & 1.6009 & 1.2488 & 1.7147 & 1.2325 & 1.7532 \\
\hline 2 & 1.2616 & 1.5553 & 1.2416 & 1.6228 & 1.2618 & 1.6607 & 1.2546 & 1.7240 & 1.3015 & 1.7853 \\
\hline 3 & 1.3301 & 1.6384 & 1.2911 & 1.6878 & 1.3429 & 1.7470 & 1.3609 & 1.8080 & 1.3718 & 1.9031 \\
\hline 4 & 1.3709 & 1.6953 & 1.3914 & 1.7649 & 1.4530 & 1.8048 & 1.4849 & 1.8462 & 1.5025 & 1.9556 \\
\hline 5 & 1.4420 & 1.7544 & 1.4710 & 1.8444 & 1.5136 & 1.8622 & 1.5528 & 1.9317 & 1.5702 & 2.0367 \\
\hline 6 & 1.5121 & 1.8337 & 1.5358 & 1.9043 & 1.5640 & 1.9213 & 1.6000 & 1.9879 & 1.6301 & 2.1015 \\
\hline 7 & 1.5902 & 1.8924 & 1.6035 & 1.9609 & 1.6369 & 1.9838 & 1.6545 & 2.0734 & 1.6804 & 2.1463 \\
\hline 8 & 1.6301 & 1.9642 & 1.6800 & 2.0605 & 1.6945 & 2.0605 & 1.7201 & 2.1699 & 1.7394 & 2.2041 \\
\hline 9 & 1.6807 & 2.0282 & 1.7550 & 2.0990 & 1.7660 & 2.1998 & 1.7728 & 2.2356 & 1.8026 & 2.2717 \\
\hline 10 & 1.7712 & 2.1199 & 1.8014 & 2.1724 & 1.8369 & 2.2695 & 1.8789 & 2.2956 & 1.9489 & 2.3339 \\
\hline
\end{tabular}

[11] J.M. Bahi and S. Contassot-Vivier. Stability of fully asynchronous discrete-time discrete-state dynamic networks.

IEEE Transactions on Neural Networks, 13(6), 1353-1363, 2002.

[12] V. Borkar and P. Varaiya. Asymptotic agreement in distributed estimation. IEEE Transactions on Automatic Control, 27(3), 650$655,1982$.

[13] J. Tsitsiklis and M. Athans. Convergence and asymptotic agreement in distributed decision problems. IEEE Transactions on Automatic Control, 29(1), 42-50, 1984.

[14] G.A. De Castro and F. Paganini. Convex synthesis of controllers for consensus. American Control Conference, 6, 4933-4938,2004.

[15] L. Fang and P.J. Antsaklis. Information consensus of asynchronous discrete-time multi-agent systems. American Control Conference, 1883-1888, 2005.

[16] F. Xiao and L. Wang. Asynchronous consensus in continuous-time multi-agent systems with switching topology and time-varying delays. IEEE Transactions on Automatic Control, 53(8), 1804-1816, 2008.

[17] M. Cao, A.S. Morse and B.D. Anderson. Agreeing asynchronously. IEEE Transactions on Automatic Control, 53(8), 1826-1838, 2008. 